\title{
BMJ Open Postoperative vascular complications in unrecognised Obstructive Sleep apnoea (POSA) study protocol: an observational cohort study in moderate-to-high risk patients undergoing non-cardiac surgery
}

\author{
Matthew T V Chan, ${ }^{1}$ Chew-Yin Wang, ${ }^{2}$ Edwin Seet, ${ }^{3}$ Stanley Tam, ${ }^{4}$ Hou-Yee Lai, ${ }^{2}$ \\ Stuart Walker, ${ }^{5}$ Timothy G Short, ${ }^{6}$ Richard Halliwell, ${ }^{7}$ Frances Chung, ${ }^{8}$ for the \\ POSA Investigators
}

To cite: Chan MTV, Wang C-Y, Seet E, et al. Postoperative vascular complications in unrecognised Obstructive Sleep apnoea (POSA) study protocol: an observational cohort study in moderate-tohigh risk patients undergoing non-cardiac surgery. BMJ Open 2014;4:e004097. doi:10.1136/bmjopen-2013004097

- Prepublication history and additional material for this paper is available online. To view these files please visit the journal online (http://dx.doi.org/10.1136/ bmjopen-2013-004097).

Received 23 September 2013 Revised 2 December 2013 Accepted 4 December 2013

CrossMark

For numbered affiliations see end of article.

Correspondence to Matthew T V Chan; mtvchan@cuhk.edu.hk

\section{ABSTRACT}

Introduction: Emerging epidemiological data suggest that obstructive sleep apnoea (OSA) is common in the general surgical population. Unfortunately, the majority of these patients are unrecognised and untreated at the time of surgery. There is substantial biological rationale to indicate that patients with unrecognised OSA are at a higher risk of postoperative vascular events. However, the extent of this morbidity is currently unknown. We have initated the postoperative vascular complications in the unrecognised obstructive sleep apnoea (POSA) study to determine the associations between OSA, nocturnal hypoxia and major postoperative vascular events in 1200 moderate-to-high risk patients undergoing major non-cardiac surgery.

Methods and analysis: The POSA study is an international prospective observational cohort study. Using a type 3 portable sleep monitoring device and ambulatory oximetry, we will quantify the severity of OSA. The primary outcome is a composite of vascular death, myocardial infarction; non-fatal cardiac arrest; stroke; pulmonary embolism; congestive heart failure and new arrhythmia within 30 days of surgery. As of November 2013, we have recruited over 700 patients from nine centres in six countries. The mean age is 68 years, the mean body mass index is $27 \mathrm{~kg} / \mathrm{m}^{2}$ and $55 \%$ of patients are men. $27.9 \%$ of patients have known coronary artery disease, over $76 \%$ have diabetes. The majority of patients underwent orthopaedic surgery $(28 \%)$ and colorectal resection $(18.5 \%)$.

Ethics and dissemination: The POSA study has received ethics approval from all study sites before patient recruitment. Informed consent will be obtained from all patients. The POSA study will determine the risk of unrecognised OSA in major non-cardiac surgery. We will publish these findings in peerreviewed journals.

Trial Registration: ClinicalTrials.gov Identifier: NCT01494181
Obstructive sleep apnoea (OSA) is the most common sleep disordered breathing affecting $17 \%$ of men and $9 \%$ of women aged between 50 and 70 years. ${ }^{1}$ In the general population, unrecognised, and therefore untreated, OSA is associated with higher risks of vascular death, myocardial infarction, stroke, heart failure and arrhythmia. ${ }^{2-7}$ This is commonly attributed to the frequent episodes of nocturnal apnoea and hypoponea that lead to oxyhaemoglobin desaturation and surges of sympathetic activity. ${ }^{8}$ In addition, OSA can increase systemic inflammation, endothelial dysfunction and platelet aggregation, ${ }^{9} 10$ which may also contribute to adverse vascular events.

General anaesthetics and potent opioid analgesics are powerful respiratory depressants. They reduce pharyngeal muscle tone and depress ventilatory response to hypoxaemia and hypercapnia. ${ }^{11}$ It is therefore not surprising that OSA might aggravate postoperative adverse cardiac events. Few studies have reported the impact of OSA on cardiac morbidity after surgery. Earlier prospective cohort studies were small trials with limited number of events. ${ }^{12}$ Subsequent analyses of several large databases, however, showed mixed results. Depending on the outcome definition, the risk of postoperative morbidity and mortality was either increased, ${ }^{13}{ }^{14}$ unchanged $^{15}$ or even paradoxically reduced in patients who were known to have or at risk for OSA. ${ }^{16}$ Interpretation of these findings is difficult because it is not clear what percentage of patients labelled as non-OSA actually had undiagnosed disease. In this regard, over $50 \%$ of patients with moderate-to-severe OSA were undiagnosed at the time of 
surgery, ${ }^{17}{ }^{18}$ and hence the results could have been biased because of group misclassification. In addition, perioperative treatment, such as non-invasive ventilation, may have also obscured the effect of OSA on the perioperative outcome.

Taken together, there is currently a lack of data documenting the extent of postoperative vascular morbidity in patients with unrecognised OSA. Therefore, a prospective observational study that accurately evaluates the presence and severity of OSA in a large group of patients, in whom a substantial number of adverse vascular events is expected to occur, will be required to establish the associations between OSA and perioperative risk. These data are important to facilitate informed decision-making about the risks of surgery and to guide perioperative management in patients with OSA. Interestingly, a clinical study to determine the associations between OSA and perioperative risk has been rated as one of the high priority areas in OSA research. ${ }^{19} 20$

\section{METHODS}

\section{Study design}

We have initiated the postoperative complications in the unrecognised obstructive sleep apnoea (POSA) studyan international prospective, observational study in 1200 patients with known, or at risk of, atherosclerotic disease undergoing major non-cardiac surgery to determine the association between OSA and postoperative vascular events. We hypothesised that patients with unrecognised OSA had a higher rate of postoperative vascular events than those who did not.

\section{Study population}

Institutional research ethics committee approval was obtained from each of the participating centres. We approach patients undergoing major elective noncardiac surgery for consideration of the study, and we recruit patients who are at moderate-to-high risk for postoperative vascular events. Box 1 shows the inclusion and exclusion criteria of the study. We excluded patients undergoing tonsillectomy, septoplasty, uvuloplasty, pharyngoplasty, tracheostomy or prolonged ( $>2$ days) mechanical ventilation of the lungs because these interventions are likely to prevent or at least modify the severity of OSA.

\section{Overnight portable sleep monitoring and pulse oximetry}

Research staff in each participating site identifies eligible patients from the daily attendance list in the preoperative clinic and the routine surgical lists for the next day. Following patient consent, all patients undergo overnight recording in the hospital surgical ward, the night before surgery, using a type 3 portable sleep monitoring device (ApneaLink, ResMed, San Diego, California, USA). Patients who are admitted on the day of surgery will have sleep monitoring performed at home in the week prior

\section{Box 1 Inclusion and exclusion criteria of the POSA study}

Patients are eligible for the study if they are:

1. Adult males and females.

2. Age $\geq 45$ years.

3. Undergoing major elective non-cardiac surgery that is expected to require a hospital stay of more than three nights.

4. At increased risk for postoperative vascular events, defined as having at least one of the following risk factors:

A. High-risk surgery (intraperitoneal, major orthopaedic or vascular surgery);

B. History of coronary artery disease;

C. History of congestive heart failure;

D. History of stroke or transient ischaemic attack;

E. Diabetes requiring insulin therapy;

F. Serum creatinine $>175 \mu \mathrm{mol} / \mathrm{L}$.

Patients will be excluded if:

1. They have a previous diagnosis of obstructive sleep apnoea or any sleep-related breathing disorder.

2. They are unwilling or physically unavailable for portable sleep monitoring within the week prior to surgery.

3. Their surgery include tonsillectomy, septoplasty, uvuloplasty, pharyngoplasty, tracheostomy or prolonged ( $>2$ days) mechanical ventilation of the lungs is anticipated after surgery.

to surgery. At bedtime, research personnel attach the device to the patient. ApneaLink contains a nasal pressure transducer to measure flow limitation and snoring. It is battery powered and records on a 16-bit signal processor, with a sampling rate of $100 \mathrm{~Hz}$. The preset internal memory is $15 \mathrm{MB}$, and thus allows continuous recording for $10 \mathrm{~h}$. In addition, we monitor oxyhaemogoblin saturation $\left(\mathrm{SpO}_{2}\right)$ using a high-resolution pulse oximeter wristwatch (PULSOX-300i, Konica Minolta Sensing, Inc, Osaka, Japan). The sampling frequency is set as $1 \mathrm{~Hz}$ with an averaging duration of $3 \mathrm{~s}$. The resolution of the pulse oximetry is $0.1 \%$. All patients breathe room air during preoperative recording.

Research staff retrieves the devices the following morning and processes the polysomnography (PSG) and oximetry data using the ApneaLink and Profox (Profox Associates, Escondido, California, USA) software, respectively. Both devices have been found to be highly sensitive and specific in determining the apnoea hypoponea index (AHI) when compared with in-laboratory or portable PSG in patients with moderate-to-severe OSA. ${ }^{21-25}$

Apnoea is defined as a decrease in airflow $\geq 90 \%$ of baseline for $>10 \mathrm{~s}$ and hypoponea as a reduction of airflow of $\geq 50 \%$ of baseline for $>10 \mathrm{~s}$ and was associated with $\geq 3 \%$ decrease in $\mathrm{SpO}_{2}$. The AHI is determined based on the recording time $(\geq 4 \mathrm{~h})$, and is the average number of apnoea and hypoponea episodes per hour. We also calculate the oxygen desaturation index (ODI) which is defined as the average number per hour of episodes of desaturation $\geq 4 \%$ from baseline and $>10 \mathrm{s.}^{25}$ OSA is diagnosed when AHI is $\geq 5$, moderate OSA is defined as AHI of 15 30 and severe OSA is when AHI $>30 .{ }^{26}$ 


\section{Perioperative care}

Before surgery, research staff interviews, examines and reviews the hospital charts of all patients to obtain information on patient characteristics. Specifically, we record data that are potential risk predictors for major perioperative vascular events according to a recently developed VISION risk model. ${ }^{27}$ Site investigators review and approve the data collected before submission to the database. In addition, we assess the patients' risk for OSA using the STOP-Bang screening tool. ${ }^{28}$

Anaesthesia and surgery are performed according to routine standard of care at each site. In order to reflect usual clinical practice and maximise generalisability, we do not control the use of postoperative analgesia, fluid management and other aspects of patient care. However, drugs used during anaesthesia, postoperative analgesia, haemodynamic data and supplemental oxygen, if required, are recorded.

\section{Patient follow-up}

During their stay in the hospital, patients are followed daily, and outcomes are recorded until discharge. After surgery, ECG and venous blood samples are collected for the measurement of plasma cardiac troponin concentrations at $6-12 \mathrm{~h}$ and daily during the first 3 days. Additional tests, such as echocardiograms and lung scans, are ordered if clinically indicated. During the first three nights after surgery, we monitor and record $\mathrm{SpO}_{2}$ using the Pulsox 300i device. Patients discharged home are contacted by phone at 30 days to ascertain whether they have experienced any adverse outcomes. If detected, we will arrange further testing and contact their hospitals and physicians-in-charge to acquire the appropriate documentation.

\section{Study outcomes}

The primary endpoint is postoperative vascular events at 30 days after surgery. It is a composite of vascular death, myocardial infarction, myocardial injury after noncardiac surgery, non-fatal cardiac arrest, revascularisation procedure, pulmonary embolism, deep venous thrombosis, new atrial fibrillation, stroke and congestive heart failure.

Secondary endpoints include the following: (1) tracheal intubation and mechanical ventilation (including use of continuous positive airway pressure ventilation) after surgery; (2) unplanned admission to the intensive care unit; (3) duration of stay in hospital and intensive care unit; (4) pneumonia and (5) infection and/or sepsis. Individual outcome definitions are listed in table 1.

\section{Outcome adjudication}

All outcomes are verified by a team of three experienced perioperative clinicians who are blinded to the AHI and STOP-Bang data collected. Only adjudicated outcomes are used for statistical analysis.
Bias control and ethical considerations

Patients and research staff who collect outcome data will be blinded to the AHI findings, STOP-Bang questionnaire and oximetry readings. Patients, attending surgeons and physicians will be given a summary report of their portable sleep study and STOP-Bang questionnaire 30 days after surgery. During this period, all patients will receive local standard of care and monitoring. Patients with abnormal AHI results will be referred to their local sleep clinic for further treatment after the conclusion of the study. This arrangement is considered appropriate and has been approved by the ethics committee in the POSA study.

\section{Statistical considerations}

We will report the proportion of patients with OSA based on their AHI findings and separately according to the severity. We will conduct a logistic regression model to examine the independent association between OSA and postoperative vascular events. The dependent variable is postoperative vascular complication within 30 days of surgery, and the independent variables are the ethnicity the VISION risk model score (a composite score of age $\geq 75$ years, history of coronary artery disease, stroke, chronic obstructive pulmonary disease, peripheral vascular disease, undergoing cancer surgery, urgent/emergent surgery and neurosurgery) ${ }^{27} 29$ and the presence of OSA (according to its severity with non-OSA as reference). In addition, we test whether a clinical screening tool, such as STOP-Bang questionnaire, could be used to predict postoperative vascular outcome. In this regression model, we replace AHI values with STOP-Bang risk score. Reliability will be determined by bootstrapping. The effect of nocturnal hypoxia (expressed as ODI) on the timing to postoperative vascular events will be determined using the Cox proportional hazard function model.

\section{Sample size}

The sample size is estimated for the logistic model, because it requires the largest number of patients to ensure model stability. Previous simulation study has demonstrated that at least 12 events per independent variable are required to produce stable estimates in logistic regression. ${ }^{30}$ Based on the findings in the VISION study, ${ }^{27} 29$ we expect a $6 \%$ event rate for postoperative vascular event in this study. Hence, at least 800 patients will be required to ensure a stable regression model. We propose to study 1200 patients to account for a Hawthorne effect where event rate may be decreased by as much as a third from $6 \%$ to $4 \%$.

\section{Study organisation and funding}

The POSA study is coordinated by the Department of Anaesthesia and Intensive Care at the Chinese University of Hong Kong. Site investigators of the trial are listed in the online supplementary appendix. ResMed has supplied the ApneaLink devices and 
Table 1 Outcome definitions

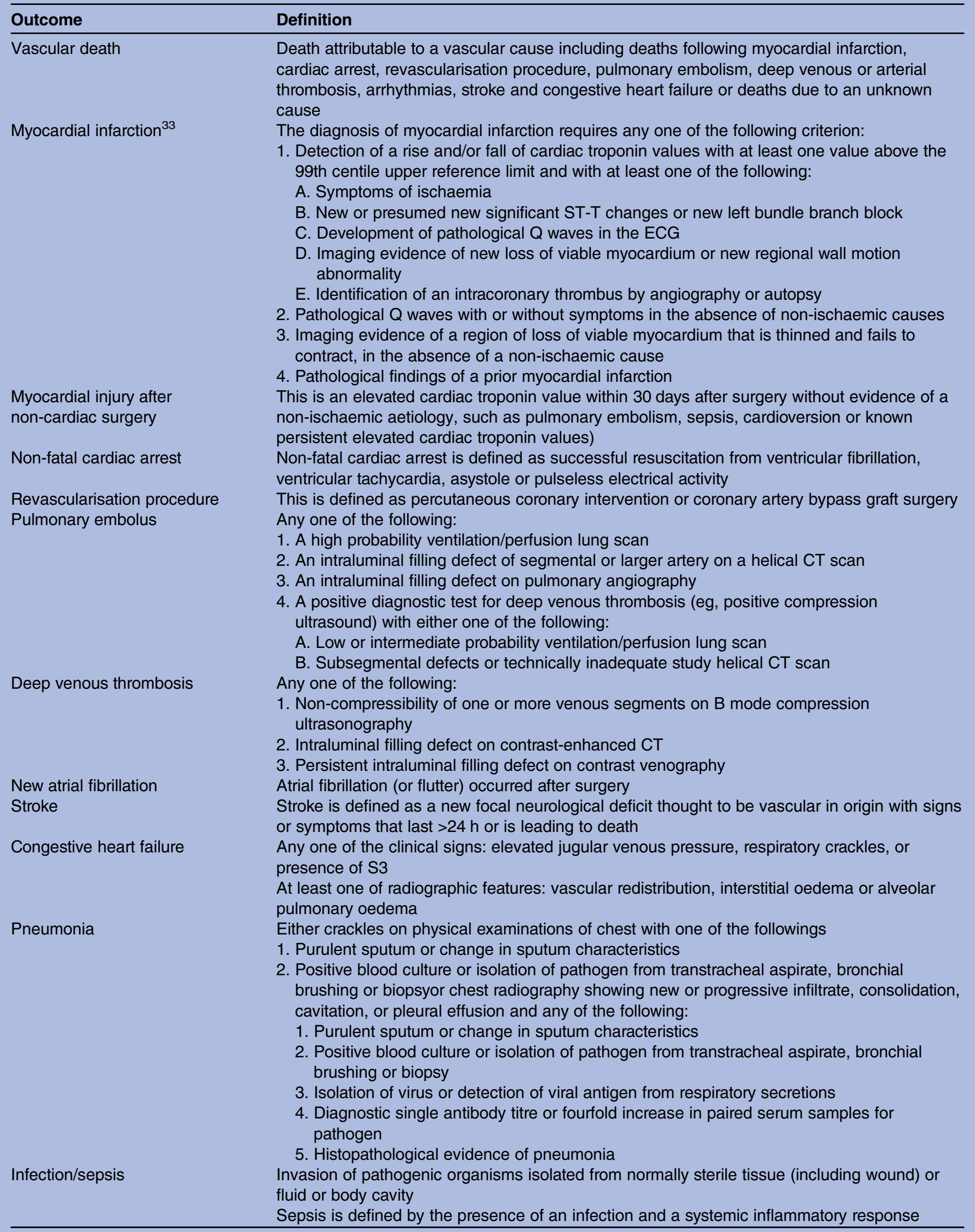

ST-T, ST-segment-T wave. 
PULSOX-300i oximeter wristwatch in all sites as an unrestricted loan. These will be returned at the end of the study.

\section{Potential limitations of the POSA study}

Given the resource constraints, it is impractical to perform standard in-laboratory attended PSG for all patients in the POSA study. We have therefore resorted to use a type 3 portable sleep monitoring device. It is known that these devices may underestimate AHI values especially in patients with mild OSA. ${ }^{31}$ Therefore, these patients may be erroneously classified as non-OSA. Nevertheless, ApneaLink measurements appeared to correlate with standard PSG. At AHI value of 5 , the sensitivity and specificity ranged between $85.4-100 \%$ and $50-100 \%$, respectively. ${ }^{21-24}$ It should be noted that we do not measure electroencephalogram in our sleep monitoring, and hence we cannot track whether the patient is asleep during measurement. We therefore standardised recordings by processing the data collected between 23:00 and 06:00, even though the patients may be awake during the period.

\section{CURRENT STATUS OF THE STUDY}

The POSA study is currently enrolling patients from nine centres in six countries. As of November 2013, we have screened 998 patients who fulfilled the POSA eligibility criteria. Among these patients, 294 were not enrolled to the study because 265 patients did not consent or their surgeons did not approve their participation. In addition, 29 patients were excluded because ApneaLink was not available $(n=2)$ or preoperative recording was not successful $(n=27)$. Currently, 704 patients are included in the POSA study and over 650 patients have completed the 30-day follow-up. Table 2 summarises the baseline characteristics and type of surgery among the first 567 patients enrolled to the study. The mean age of patients was 68 years, $55 \%$ were male, $27.9 \%$ had a history of known coronary artery disease and $75.8 \%$ had three risk factors for atherosclerosis. The majority of patients underwent orthopaedic $(28 \%)$, general $(31 \%)$, urological $(8.6 \%)$ and vascular $(6.9 \%)$ surgery.

\section{DISCUSSION}

OSA is a common condition that is often unrecognised, undiagnosed and associated with significant morbidity and mortality. The POSA study will inform clinicians about the magnitude and severity of unrecognised OSA in the surgical population in different countries. More importantly, it will determine the risks of postoperative vascular events in patients with unrecognised and untreated OSA. Specifically, it will facilitate surgeons and patients in making decisions and will guide perioperative patient management. Furthermore, the findings will provide insights into the possible harmful effect of nocturnal hypoxia. This knowledge will inform the possible mechanisms of postoperative vascular events and should

\begin{tabular}{lc} 
Table 2 Baseline characteristics & \\
\hline Variables & Values \\
\hline Number of patients & 567 \\
Age (year) & $68 \pm 10$ \\
Weight (kg) & $70 \pm 16$ \\
Height $(\mathrm{cm})$ & $161 \pm 10$ \\
BMI (kg/m ${ }^{2}$ ) & $27.0 \pm 5.5$ \\
Male, $\mathrm{n}(\%)$ & $312(55)$ \\
Ethnicity, $\mathrm{n}(\%)$ & \\
Chinese & $272(48.1)$ \\
Malay/lndian & $187(32.9)$ \\
Caucasian & $108(18.9)$ \\
ASA physical status, $\mathrm{n}(\%)$ & \\
ASA II & $336(59.3)$ \\
ASA III & $217(38.3)$ \\
ASA IV & $14(2.4)$ \\
Comorbidity, $\mathrm{n}$ (\%) & \\
Hypertension & $487(85.9)$ \\
Coronary artery disease & $158(27.9)$ \\
Congestive heart failure & $21(3.7)$ \\
Peripheral vascular disease & $51(9)$ \\
Cerebrovascular disease & $96(16.9)$ \\
Diabetes mellitus & $435(76.7)$ \\
Renal impairment & $51(9)$ \\
Current smokers & $198(34.9)$ \\
Surgical types, $\mathrm{n}(\%)$ & $105(18.5)$ \\
Colorectal surgery & $71(12.5)$ \\
Liver surgery & $49(8.6)$ \\
Genitourinary surgery & $159(28)$ \\
Orthopaedics & $34(6)$ \\
Spine & $39(6.9)$ \\
Vascular surgery & $110(19.4)$ \\
Others & $101(17.8)$ \\
Minimally invasive surgery, $\mathrm{n}(\%)$ & $2.25 \pm 2.39$ \\
Duration of surgery (hours) & \\
\hline Values are number $(\%)$ or mean \pm SDs. & \\
ASA, American Society of Anaesthesiologists; BMI, body mass \\
index. & \\
& \\
\hline &
\end{tabular}

stimulate future research into the prevention of this harm. Over 200 million patients undergo surgery each year, ${ }^{32}$ many of them have significant OSA, we believe any improvement in postoperative outcome should have major implications for healthcare delivery.

\section{Author affiliations}

${ }^{1}$ Department of Anaesthesia and Intensive Care, The Chinese University of Hong Kong, Prince of Wales Hospital, Hong Kong, People's Republic of China ${ }^{2}$ Faculty of Medicine, Department of Anaesthesiology, University Malaya, Kuala Lumpur, Malaysia

${ }^{3}$ Department of Anaesthesia, Khoo Teck Puat Hospital, Singapore, Singapore ${ }^{4}$ Department of Anesthesia, Scarborough General Hospital, Ontario, Canada ${ }^{5}$ Department of Anaesthesia, Middlemore Hospital, Manukau City, New Zealand

${ }^{6}$ Department of Anaesthesia, Auckland City Hospital, Auckland, New Zealand ${ }^{7}$ Department of Anaesthesia, Westmead Hospital, Sydney, Australia

${ }^{8}$ Department of Anesthesia, Toronto Western Hospital, University Health Network, University of Toronto, Ontario, Canada

Acknowledgements The authors would like to thank all the research assistants and ward staff for their dedications and ResMed for providing research equipment to the POSA study. 
Collaborators POSA investigators: David Hui, Gordon Choi, Tony Gin, Matthew Tsang, Beaker Fung, Angela Miu, Alex Lee, Eleanoir FF Chew, Carolyn CW Yim, Alvin SB Tan, Su-Yin Loo, Simon MT Hui, Peter Liao, Sohail Iqbal, Joyce Tai, Sue Olliff, Ivan Bergman and Melanie Speer.

Contributors All authors provided intellectual input into the editing of the manuscript and preparation for publication.

Funding The work is supported by Health and Medical Research Fund, Food and Health Bureau, Hong Kong SAR, Competitive Grant from the University of Toronto; High Impact Research Grant UM.C/625/1/HIR/067 from the University of Malaya and Innovation Grant from Alexandra Health, Singapore.

Competing interests None.

Patient consent Obtained.

Ethics approval Joint CUHK-NTEC Clinical Research Ethics Committee, Hong Kong.

Provenance and peer review Not commissioned; externally peer reviewed.

Open Access This is an Open Access article distributed in accordance with the Creative Commons Attribution Non Commercial (CC BY-NC 3.0) license, which permits others to distribute, remix, adapt, build upon this work noncommercially, and license their derivative works on different terms, provided the original work is properly cited and the use is non-commercial. See: http:// creativecommons.org/licenses/by-nc/3.0/

\section{REFERENCES}

1. Peppard PE, Young T, Barnet JH, et al. Increased prevalence of sleep-disordered breathing in adults. Am J Epidemiol 2013;177:1006-14.

2. Young T, Finn L, Peppard PE, et al. Sleep disordered breathing and mortality: eighteen-year follow-up of the Wisconsin sleep cohort. Sleep 2008;31:1071-8.

3. Marshall NS, Wong KKH, Liu PY, et al. Sleep apnea as an independent risk factor for all-cause mortality: the Busselton Health Study. Sleep 2008;31:1079-85.

4. Punjabi NM, Newman AB, Young TB, et al. Sleep-disordered breathing and cardiovascular disease: an outcome-based definition of hypopneas. Am J Respir Crit Care Med 2008;177:1150-5.

5. Mehra R, Benjamin EJ, Shahar E, et al. Association of nocturnal arrhythmias with sleep-disordered breathing: the Sleep Heart Health Study. Am J Respir Crit Care Med 2006;173:910-16.

6. Selim B, Won C, Yaggi HK. Cardiovascular consequences of sleep apnea. Clin Chest Med 2010;31:203-20.

7. Kendzerska T, Mollayeva T, Gershon AS, et al. Untreated obstructive sleep apnea and the risk for serious long-term adverse outcomes: a systematic review. Sleep Med Rev 2013;18:49-59.

8. Gami AS, Olson EJ, Shen WK, et al. Obstructive sleep apnea and the risk of sudden cardiac death: a longitudinal study of 10,701 adults. J Am Coll Cardiol 2013;62:610-16.

9. Barcelo A, Pierola J, de la Pena M, et al. Day-night variations in endothelial dysfunction markers and haemostatic factors in sleep apnoea. Eur Respir J 2012;39:913-18.

10. Barcelo A, Pierola J, de la Pena M, et al. Impaired circadian variation of platelet activity in patients with sleep apnea. Sleep Breath 2012;16:355-60.

11. Chau EH, Lam D, Wong J, et al. Obesity hypoventilation syndrome: a review of epidemiology, pathophysiology, and perioperative considerations. Anesthesiology 2012;117:188-205.

12. Kaw R, Chung F, Pasupuleti V, et al. Meta-analysis of the association between obstructive sleep apnoea and postoperative outcome. Br J Anaesth 2012;109:897-906.
13. Kaw R, Pasupuleti V, Walker E, et al. Postoperative complications in patients with obstructive sleep apnea. Chest 2012;141:436-41.

14. Memtsoudis S, Liu SS, Ma Y, et al. Perioperative pulmonary outcomes in patients with sleep apnea after noncardiac surgery. Anesth Analg 2011;112:113-21.

15. Lockhart EM, Willingham MD, Abdallah AB, et al. Obstructive sleep apnea screening and postoperative mortality in a large surgical cohort. Sleep Med 2013;14:407-15.

16. Mokhlesi B, Hovda MD, Vekhter B, et al. Sleep-disordered breathing and postoperative outcomes after elective surgery: analysis of the nationwide inpatient sample. Chest 2013;144:903-14.

17. Singh M, Liao P, Kobah S, et al. Proportion of surgical patients with undiagnosed obstructive sleep apnoea. $\mathrm{Br} J$ Anaesth 2013;110:629-36.

18. Finkel KJ, Searleman AC, Tymkew H, et al. Prevalence of undiagnosed obstructive sleep apnea among adult surgical patients in an academic medical center. Sleep Med 2009;10:753-8.

19. Chung F, Hillman D, Lydic R. Sleep medicine and anesthesia: a new horizon for anesthesiologists. Anesthesiology 2011;114:1261-2.

20. Patel K, Moorthy D, Chan JA, et al. High priority future research needs for obstructive sleep apnea diagnosis and treatment. J Clin Sleep Med 2013;9:395-402.

21. $\mathrm{Ng}$ SSS, Chan TO, To KW, et al. Validation of a portable recording device (ApneaLink) for identifying patients with suspected obstructive sleep apnoea syndrome. Int Med J 2009;39:757-62.

22. Erman MK, Stewart D, Einhorn D, et al. Validation of the ApneaLink for the screening of sleep apnea: a novel and simple single-channel recording device. J Clin Sleep Med 2007;3:387-92.

23. Crowley KE, Rajaratnam SM, Shea SA, et al. Evaluation of a single-channel nasal pressure device to assess obstructive sleep apnea risk in laboratory and home environments. J Clin Sleep Med 2013;9:109-16.

24. Chen $\mathrm{H}$, Lowe $\mathrm{A}$, Bai $\mathrm{Y}$, et al. Evaluation of a portable recording device (ApneaLink ${ }^{\mathrm{TM}}$ ) for case selection of obstructive sleep apnea. Sleep Breath 2009;13:213-19.

25. Chung $\mathrm{F}$, Liao $\mathrm{P}$, Elsaid $\mathrm{H}$, et al. Oxygen desaturation index from nocturnal oximetry: a sensitive and specific tool to detect sleep-disordered breathing in surgical patients. Anesth Analg 2012;114:993-1000.

26. Iber C, Ancoli-Israel S, Quan SF, et al. The AASM manual for the scoring of sleep and associated events: rules, terminology and technical specifications. 1st edn. Westchester, IL: American Academy of Sleep Medicine, 2007

27. Devereaux PJ, Chan MTV, Alonso-Coello P, et al. Association between postoperative troponin levels and 30-day mortality among patients undergoing noncardiac surgery. JAMA 2012;307:2295-304.

28. Chung F, Yegneswaran B, Liao P, et al. STOP questionnaire: a tool to screen patients for obstructive sleep apnea. Anesthesiology 2008;108:812-21.

29. Botto F, Alonso-Coello P, Chan MTV, et al. Myocardial injury after noncardiac surgery: a large international prospective cohort study establishing diagnostic criteria, characteristics, predictors, and 30-day outcomes. Anesthesiology, Lippincott Williams \& Wilkins, 2013. In press.

30. Babyak MA. What you see may not be what you get: a brief, nontechnical introduction to overfitting in regression-type models. Psychosom Med 2004;66:411-21.

31. Chesson AL, Berry RB, Pack A. Practice parameters for the use of portable monitoring devices in the investigation of suspected obstructive sleep apnea in adults. Sleep 2003;26:907-13.

32. Weiser TG, Regenbogen SE, Thompson KD, et al. An estimation of the global volume of surgery: a modelling strategy based on available data. Lancet 2008;372:139-44.

33. Thygesen K, Alpert JS, Jaffe AS, et al. Third universal definition of myocardial infarction. Circulation 2012;126:2020-35. 